\title{
Effects of imaging gradients in sequences with varying longitudinal storage time-Case of diffusion exchange imaging
}

\author{
Lasic, Samo; Lundell, Henrik; Topgaard, Daniel; Dyrby, Tim Bjørn
}

Published in:

Magnetic Resonance in Medicine

Link to article, DOI:

$10.1002 / \mathrm{mrm} .26856$

Publication date:

2017

Document Version

Peer reviewed version

Link back to DTU Orbit

Citation (APA):

Lasic, S., Lundell, H., Topgaard, D., \& Dyrby, T. B. (2017). Effects of imaging gradients in sequences with varying longitudinal storage time-Case of diffusion exchange imaging. Magnetic Resonance in Medicine, 79(4), 2228-2235. https://doi.org/10.1002/mrm.26856

\section{General rights}

Copyright and moral rights for the publications made accessible in the public portal are retained by the authors and/or other copyright owners and it is a condition of accessing publications that users recognise and abide by the legal requirements associated with these rights.

- Users may download and print one copy of any publication from the public portal for the purpose of private study or research.

- You may not further distribute the material or use it for any profit-making activity or commercial gain

- You may freely distribute the URL identifying the publication in the public portal 


\title{
Effects of Imaging Gradients in Sequences With Varying Longitudinal Storage Time-Case of Diffusion Exchange Imaging
}

\author{
Samo Lasič $\odot,{ }^{1,2 *}$ Henrik Lundell, ${ }^{1}$ Daniel Topgaard $\odot,{ }^{3}$ and Tim B. Dyrby ${ }^{1,4}$
}

Purpose: To illustrate the potential bias caused by imaging gradients in correlation MRI sequences using longitudinal magnetization storage (LS) and examine the case of filter exchange imaging (FEXI) yielding maps of the apparent exchange rate (AXR).

Methods: The effects of imaging gradients in FEXI were observed on yeast cells. To analyze the AXR bias, signal evolution was calculated by applying matrix exponential operators.

Results: A sharp threshold for the slice thickness was identified, below which the AXR is increasingly underestimated. The bias can be understood in terms of an extended low-pass diffusion filtering during the LS interval, which is more pronounced at lower exchange rates. For a total exchange rate constant larger than $1 \mathrm{~s}^{-1}$, the AXR bias is expected to be negligible when slices thicker than $2.5 \mathrm{~mm}$ are used.

Conclusion: In correlation experiments like FEXI, relying on LS with variable duration, imaging gradients may cause disrupting effects that cannot be easily mitigated and should be carefully considered for unbiased results. In typical clinical applications of FEXI, the imaging gradients are expected to cause a negligible AXR bias. However, the AXR bias may be significant in preclinical settings or whenever thin imaging slices are used. Magn Reson Med 000:000-000, 2017. () 2017 International Society for Magnetic Resonance in Medicine.

Key words: slice; crusher; longitudinal storage; double diffusion encoding; mixing time; FEXI

\section{INTRODUCTION}

Magnetic resonance techniques that are specifically sensitive to cell membrane permeability and allow for non-invasive

${ }^{1}$ Danish Research Centre for Magnetic Resonance, Centre for Functional and Diagnostic Imaging and Research, Copenhagen University Hospital Hvidovre, Hvidovre, Copenhagen, Denmark.

${ }^{2}$ CR Development AB, Lund, Sweden.

${ }^{3}$ Physical Chemistry, Lund University, Lund, Sweden.

${ }^{4}$ Department of Applied Mathematics and Computer Science, Technical University of Denmark, Kongens Lyngby, Denmark.

Grant sponsor: VINNMER Marie Curie Industry Outgoing Grant; Grant number: 013-04350; Grant sponsor: Swedish Foundation for Strategic Research; Grant number: AM13-0090; Grant sponsor: Swedish Research Council; Grant number: 2014-3910; Grant sponsor: CR Development AB (Lund, Sweden); Grant sponsor: Danish Multiple Sclerosis Society; Grant number: A-27996; Grant sponsor: Capital Region Research Foundation for Healthcare; Grant sponsor: Danish Research Council for Independent Research, Production and Technology; Grant number: 4093-00280A and 4093-00280B.

*Correspondence to: Samo Lasič, Ph.D., CR Development AB, Naturvetarevägen 60, 22241 Lund, Sweden. E-mail: samo@crdev.se.

D.T. is co-owner of the company CR Development AB (Lund, Sweden), holding patents related to the described method.

Received 15 March 2017; revised 28 June 2017; accepted 7 July 2017

DOI 10.1002/mrm.26856

Published online 00 Month 2017 in Wiley Online Library (wileyonlinelibrary. com).

(C) 2017 International Society for Magnetic Resonance in Medicine mapping of molecular exchange are based on the correlation principle $(1,2)$, where consecutive signal weighting is applied within a single pulse sequence. Such experiments rely on e.g., differences in relaxation rates (3) or apparent diffusivities (4) between the exchanging compartments. Diffusion exchange spectroscopy (DEXSY) (4) can be used to quantify the rate of exchange between compartments with different apparent diffusivities. The filter exchange spectroscopy (FEXSY) (5) and the filter exchange imaging (FEXI) (6) are sparse sampling protocols (1) based on DEXSY, which allow quantifying exchange within significantly reduced experimental times. While FEXSY provides the exchange rate constants for a two-compartment system, e.g., a cell suspension [5], the clinically feasible FEXI protocol can map the apparent exchange rate (AXR) (6). After initial experiments on the human brain in vivo (7), FEXI was applied on a fixed monkey brain to investigate the effect of macroscopic tissue anisotropy on AXR (8). More recently, the FEXI protocol was optimized for minimal measurement variance in AXR and used to characterize human brain tumors in vivo (9). AXR was measured in suspensions of cancer breast cells and in breast tumors in vivo (10). FEXI also shows promising results in detection of urea transporters (11). DEXSY, FEXSY, and FEXI protocols use a double diffusion encoding (DDE) sequence (12). A recent study demonstrated that, with present clinical scanners, FEXI is indeed advantageous compared to a protocol using single diffusion encoding (SDE) sequence (13).

The key component of the above-mentioned exchange experiments based on DDE is longitudinal storage (LS) with varying duration. LS is probably best known as an essential building block of the stimulated echo (STE) sequence, often used in applications with short $\mathrm{T}_{2}$, e.g., at higher magnetic field strengths. LS consists of two $90^{\circ}$ radio frequency (RF) pulses separated by the "mixing time" during which magnetization is "stored" parallel to the main magnetic field (14). In exchange experiments, the term "mixing block" is commonly used, reflecting the ability of spins to mix or move between different compartments.

In spectroscopic experiments $(4,5,15)$, phase cycling of the receiver and the $\mathrm{LS} 90^{\circ} \mathrm{RF}$ pulses allows selecting the desired coherence pathway $(14,16)$. In imaging experiments (7-10), phase cycling can be replaced by "crusher" gradients, straddling the $90^{\circ}$ pulses of the LS block. Unfortunately, slice and crusher gradients (16), herein together called the "butterfly" gradients, may cause undesired diffusion weighting during the LS block (see Fig. 1). The butterfly gradients could also disrupt the experimental design when single diffusion-weighted STE is used, e.g., in high angular resolution diffusion 


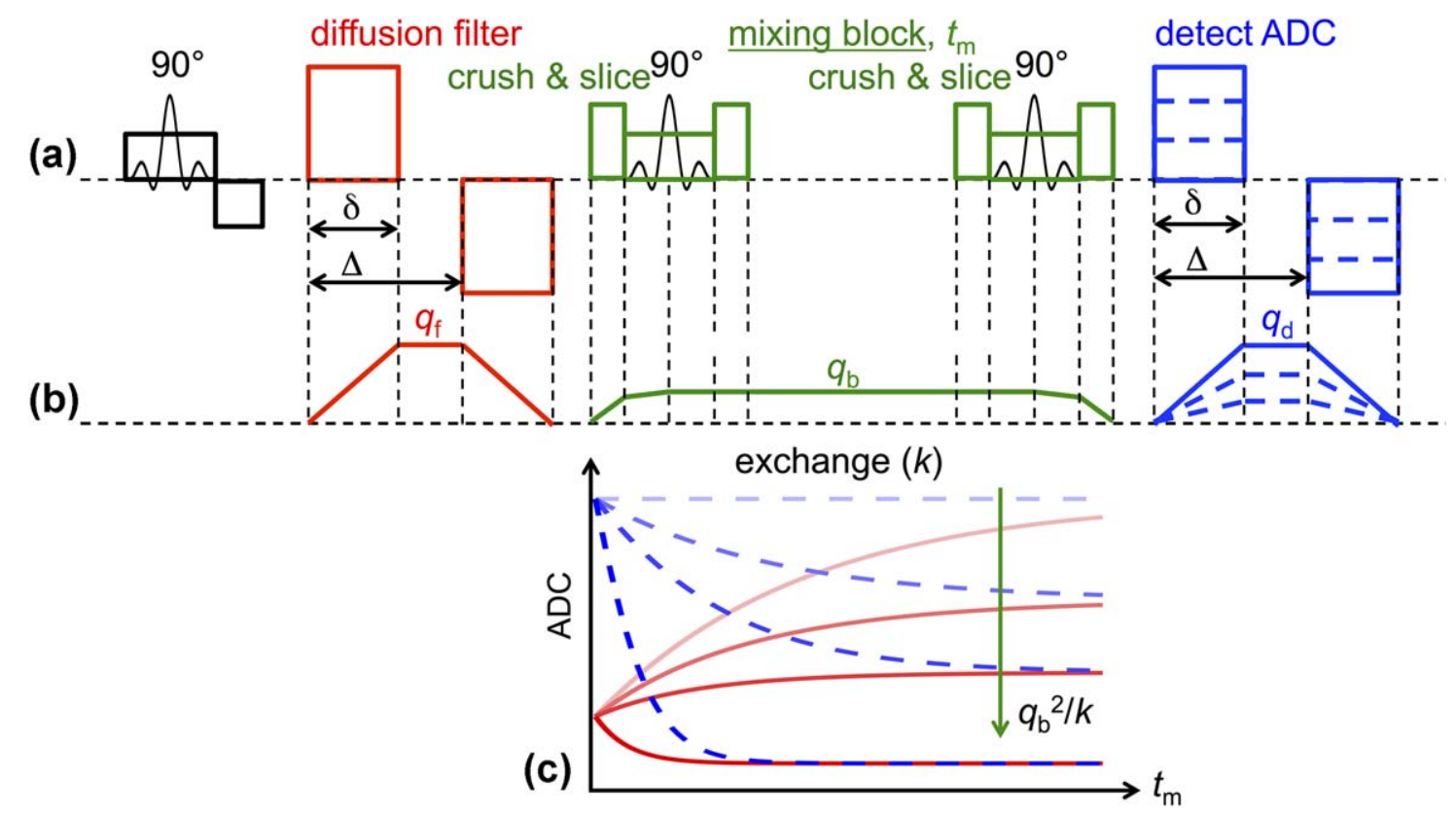

FIG. 1. FEXI pulse sequence with imaging gradients. (a) The pulse sequence consists of two diffusion-encoding blocks (red and blue) separated by the mixing block (green). Shown are the $90^{\circ} \mathrm{RF}$ pulses and the rectangular gradient pulses. The duration of the mixing block $t_{\mathrm{m}}$ is varied, whereas the gradient pulse duration $\delta$ and spacing $\Delta$ are kept constant in the "filter" (red) and "detection" (blue) blocks. Additional imaging gradients are applied before and during longitudinal storage RF pulses (crush and slice shown in green). (b) The dephasing parameter $q(t)$ for the FEXI sequence. Note that only the imaging gradients applied before and after the longitudinal storage interval contribute to signal dephasing. (c) Variation of the apparent diffusion coefficient (ADC) during mixing time $t_{\mathrm{m}}$. Red solid lines and blue dashed lines correspond to the sequence with and without the first encoding block (filter), respectively. The asymptotic value of $\mathrm{ADC}$ at long $t_{\mathrm{m}}$ decreases with the ratio of $q_{b}^{2} / k$ according to Eq. [5].

imaging experiments. Because in this case, the diffusion weighting is desired during the LS interval, the effects of butterfly gradients can be corrected in the analysis or compensated in the acquisition $(17,18)$. On the other hand, in experiments that rely on varying the LS time, during which diffusion weighting is not desired, the butterfly gradients may introduce a bias that cannot be easily mitigated.

The analysis presented here applies to any correlation pulse sequence using LS with variable mixing time. As an example, we examine the bias caused by butterfly gradients in FEXI and their possible confounding effect on the measurement of the AXR.

\section{THEORY}

\section{Two-Compartment Signal Model in FEXI}

Exchange between two compartments with different apparent diffusivities can be described according to the first order kinetics $(19,20)$. For a single diffusion-weighted sequence, assuming time-independent diffusion, the result is known as the Kärger model $(21,22)$. Modified models can be used to account for restricted diffusion effects in the regime of slow exchange (23-25) or for considering exchange during diffusion-encoding gradient pulses (26). For sequences with multiple encoding blocks, the evolution of signals from compartments 1 and $2, S_{1}$ and $S_{2}$, is given by a composite of sequential operators associated with each encoding block. This representation of the signal evolution, common also in NMR spectroscopy (27), proves convenient to analyze the effects of butterfly gradients in FEXI.
In the FEXI sequence (Fig. 1a), the signal evolution can be decomposed into three parts: the diffusion filter, the mixing interval $t_{\mathrm{m}}$, and the diffusion detection block. After a gradient pulse, the dephasing parameter $q(t)$, shown in Figure $1 \mathrm{~b}$, reaches the amplitude $q=\gamma G \delta$, where $\gamma$ is the nuclear gyromagnetic ratio, $G$ is the gradient pulse amplitude, and $\delta$ its duration. With $q_{\mathrm{b}}$, we denote the undesired dephasing because of the butterfly gradients, colored green in Figure 1. The diffusion weighting is given by $b=q^{2} t$, where $t$ is the dephasing time. For the diffusion encoding block with pulse separation $\Delta, t$ is the effective diffusion time given by $t_{\mathrm{d}}=\Delta-\delta / 3$, while for the mixing block, $t$ is approximately equal to the mixing time $t_{\mathrm{m}}$.

In the following, we will ignore diffusion and exchange effects during gradient pulses and assume equal relaxation rates for the two compartments. For simplicity, we assume time-independent diffusion in each compartment; however, the analysis can easily be adapted to account for time-dependent diffusion effects (see the Discussion for further details). The evolution of signals $\mathbf{S}^{\mathrm{T}}=\left(S_{1}, S_{2}\right)$ is given by the composite of operators $\mathbf{O}$ as

$$
\mathbf{S}=S_{0} \mathbf{O}_{\mathrm{d}} \times \mathbf{O}_{\mathrm{m}} \times \mathbf{O}_{\mathrm{f}} \mathbf{f}
$$

where $S_{0}$ is the relaxation-weighted signal without diffusion encoding, $\mathbf{f}^{\mathrm{T}}=\left(f_{1}, 1-f_{1}\right)$ are the signal fractions, and the operator subscripts " $\mathrm{d}$," "m," and "f" refer to the diffusion detection, mixing, and filter blocks, respectively. All three operators can be expressed in a common form of matrix exponentials, 


$$
\mathbf{O}=\exp \left[-\left(q^{2} \mathbf{D}+\mathbf{K}\right) t\right]
$$

where the dephasing magnitudes $q_{\mathrm{d}}, q_{\mathrm{b}}$, and $q_{\mathrm{f}}$ and time intervals $t_{\mathrm{d}}, t_{\mathrm{m}}$, and $t_{\mathrm{d}}$ apply to the detection, mixing, and filter blocks, respectively. The exchange matrix is defined as

$$
\mathbf{K}=\left(\begin{array}{cc}
k_{12} & -k_{21} \\
-k_{12} & k_{21}
\end{array}\right)
$$

where $k_{12}$ and $k_{21}$ are the forward/backward exchange rate constants fulfilling the equilibrium condition $\mathbf{K f}=0$ (28), and the diffusion matrix

$$
\mathbf{D}=\left(\begin{array}{cc}
D_{1} & 0 \\
0 & D_{2}
\end{array}\right)
$$

holds diffusion coefficients $D_{1,2}$ for the two compartments.

\section{Apparent Diffusion Coefficient}

The total signal attenuation $S(b)$ is given by the sum of the two components in Eq. [1], $S(b)=\mathbf{S}(1)+\mathbf{S}(2)$. The apparent diffusion coefficient (ADC) can be evaluated from $\frac{\partial}{\partial b} \ln \frac{S(b)}{S_{0}}$ in the limit of small $b$ (6). At long mixing times, $t_{\mathrm{m}} \rightarrow \infty$, the effect of the diffusion filter is lost as a result of exchange (6) and the filtered and non-filtered ADC values converge, as shown in Figure 1c. The asymptotic $\mathrm{ADC}_{\infty}$ can therefore be calculated by omitting the filter (i.e., by setting $\mathbf{O}_{\mathrm{f}}$ equal to the identity matrix in Eq. [1]). Taylor expansion of $\mathrm{ADC}_{\infty}$ for small butterfly gradient contribution (i.e., when $q_{\mathrm{b}} \rightarrow 0$ ), yields

$$
\begin{aligned}
\mathrm{ADC}_{\infty}= & \mathrm{ADC}^{\mathrm{eq}}-f_{1} f_{2}\left(D_{1}-D_{2}\right)^{2} \frac{q_{\mathrm{b}}^{2}}{k} \\
& +f_{1} f_{2}\left(1-2 f_{1}\right)\left(D_{1}-D_{2}\right)^{3}\left(\frac{q_{\mathrm{b}}^{2}}{k}\right)^{2} \\
& -f_{1} f_{2}\left(1-5 f_{1} f_{2}\right)\left(D_{1}-D_{2}\right)^{4}\left(\frac{q_{\mathrm{b}}^{2}}{k}\right)^{3}+\ldots,
\end{aligned}
$$

where $k=k_{12}+k_{21}$ is the total exchange rate constant and $f_{2}=1-f_{1}$. Note that in the absence of butterfly gradients, when $q_{\mathrm{b}}=0$, Eq. [5] yields, as expected, the weighted average $A D C^{\mathrm{eq}}=f_{1} D_{1}+f_{2} D_{2}$. We see that the $\mathrm{ADC}_{\infty}$ is modulated by the signal fraction and by the difference $D_{1}-D_{2}$, but most importantly, it is determined by the ratio $q_{\mathrm{b}}^{2} / k$. This means that the $\mathrm{ADC}_{\infty}$ modulation caused by the unwanted $q_{\mathrm{b}}^{2}$ contribution is more pronounced for lower values of $k$.

\section{Slice Thickness and the Minimum Strength of the Butterfly Gradients}

The minimum strength of the butterfly gradients required by an imaging sequence depends on the slice thickness $\Delta z$ (16). To select the desired echo pathway, a minimum phase shift of $4 \pi$ across the slice is recommended (16). This sets the minimum required crusher dephasing of $4 \pi / \Delta z$. The RF spectral bandwidth $\Delta f$ is related to the slice gradient magnitude $G_{\mathrm{s}}$ and slice thickness as
$2 \pi \Delta f=\gamma G_{s} \Delta z$. For simplicity, we assume that the slice gradients produce a dephasing magnitude, which is given by half-pulse duration $\delta_{\mathrm{s}} / 2$ as $\gamma G_{\mathrm{s}} \delta_{\mathrm{s}} / 2$. Together, the minimum dephasing magnitude for the butterfly gradients (crusher and slice) is therefore given by

$$
q_{\mathrm{b}, \min }=\frac{4 \pi+\pi \Delta f \delta_{\mathrm{s}}}{\Delta z} .
$$

Using minimum crusher gradients, the fractional contribution of crusher versus slice gradients in Eq. [6] is given by $4 /\left(\Delta f \delta_{s}\right)$, which is approximately $2 / 3$ in our experiments. Note that in practice the slice selection gradient is expected to produce dephasing that varies over the slice thickness. A more rigorous study of diffusion weighting during slice-selective RF pulses, which also applies to adiabatic pulses generating non-linear magnetization phase across the slice, is provided by $(29,30)$.

\section{METHODS}

\section{Experiments}

Experiments were carried out to qualitatively verify the expected effects of butterfly gradients in FEXI. Fresh baker's yeast mixed with tap water (3:1 weight ratio) was measured at room temperature on a preclinical Agilent 4.7 T MRI scanner similar as in (8). FEXI was carried out with: repetition time $=2.5 \mathrm{~s}, \delta / \Delta=5 / 10 \mathrm{~ms}$, seven linearly spaced $t_{\mathrm{m}}$ between 10 and $300 \mathrm{~ms}$, diffusion weighting of the filter block, $b_{\mathrm{f}}=0$ and $2381 \mathrm{~s} / \mathrm{mm}^{2}$ and the detection block, $b=45,50,61,93,192,489$, and $1387 \mathrm{~s} /$ $\mathrm{mm}^{2}$. The detection block $b$ values result from independently varying the gradient pulse amplitudes for each $t_{\mathrm{m}}$. Crusher gradients with amplitudes 0, 3.3, 7.2, 16.2, 36, 80, 180, and $400 \mathrm{mT} / \mathrm{m}$ and duration $1 \mathrm{~ms}$ were applied in the same direction as the slice gradients. Three 3-mm slices were excited with sinc modulated RF pulses $\left(\delta_{\mathrm{s}}=2 \mathrm{~ms}\right)$ with bandwidth, $\Delta f=2972 \mathrm{~Hz}$. The dephasing magnitudes of the crusher gradients were logarithmically spaced in the range of 0 to $107 \mathrm{~mm}^{-1}$ resulting in total butterfly dephasing magnitudes $q_{\mathrm{b}}$ in the range of 6.22 to $113 \mathrm{~mm}^{-1}$. Note that for the slice thickness of $3 \mathrm{~mm}$, the minimum required crusher dephasing magnitude is $4.2 \mathrm{~mm}^{-1}$. The mean values and confidence intervals for $\operatorname{ADC}\left(t_{\mathrm{m}}\right)$ shown in Figure 2 are a result of a voxel-based signal analysis from 216 voxels across all three slices.

\section{Simulations}

Simulations were carried out to illustrate the effects of butterfly gradients in FEXI and estimate the relative bias of AXR as a function of the imaging slice thickness. Noiseless synthetic data were generated according to the two-compartment exchange model given by Eqs. [1-4]. Filtered and non-filtered signal evolutions were calculated by engaging or disengaging the $\mathbf{O}_{\mathrm{f}}$ operator in Eq. [1]. The sequence parameters were: $b=0,3,6.5,13.9,30$, 64.6, $139,300 \mathrm{~s} / \mathrm{mm}^{2}, \delta / \Delta=5 / 10 \mathrm{~ms}$, seven linearly spaced $t_{\mathrm{m}}$ between 3 and $300 \mathrm{~ms}$. The filter $b$ value was switched between 0 and $b_{\mathrm{f}}$, which was $1000 \mathrm{~s} / \mathrm{mm}^{2}$ (Figs. 3 and 4a) or varied (Figs. 4b and 5). Data were generated for a variable slice thickness corresponding to 


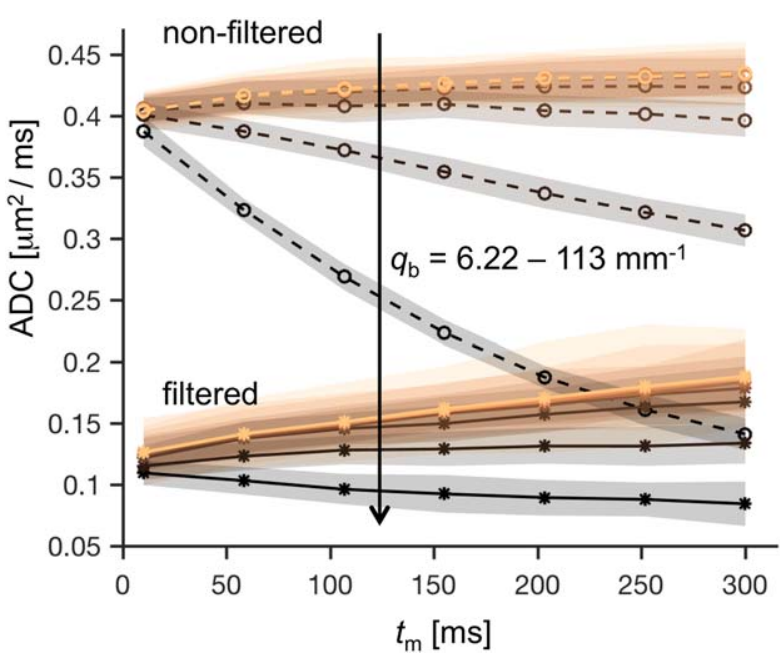

FIG. 2. Experimental results from yeast cell suspension using FEXI and crusher gradients with varying strength. Shown are the $\operatorname{ADC}\left(t_{m}\right)$, for experiments without/with diffusion filter $\left(b_{f}=0 / 2381\right.$ $\mathrm{s} / \mathrm{mm}^{2}$, circle/asterisk markers connected with dashed/solid lines). The crusher gradient was increased in eight steps (color coded from light brown to black). The lines represent mean values and the shaded areas represent confidence intervals of $68.3 \%$ from 216 voxels across three slices. The arrow indicates the increasing dephasing as a result of butterfly gradients $q_{\mathrm{b}}$.

the minimum dephasing of the butterfly gradients $q_{\mathrm{b}}$ according to Eq. [6]. The parameters $\Delta f$ and $\delta_{\mathrm{s}}$ were identical to those used in experiments. A two-compartment system with $f_{1}=0.5$ was considered. The apparent diffusivities of $D_{1}=1.3 \mu \mathrm{m}^{2} / \mathrm{ms}$ and $D_{2}=0.077 \mu \mathrm{m}^{2} / \mathrm{ms}$, yielding $D^{\mathrm{eq}}=0.69 \mu \mathrm{m}^{2} / \mathrm{ms}$ (Figs. 3 and $4 \mathrm{~b}$ ), were chosen to mimic yeast cell suspension data (31). $D_{2}$ was calculated for a spherical restriction (32) with diameter of $5 \mu \mathrm{m}$ and the intrinsic diffusivity of $0.65 \mu \mathrm{m}^{2} / \mathrm{ms}$ corresponding to yeast suspension data (31). The diffusivities $D_{1}$ and $D_{2}$ were proportionally scaled to yield variable $D^{\text {eq }}$ (Figs. 4 a, $4 \mathrm{~b}$, and 5). A range of ten linearly spaced total exchange rate constants, $k=1-10 \mathrm{~s}^{-1}$, was used (Fig. 3) or fixed to $1 \mathrm{~s}^{-1}$ (Figs. 4 and 5). These exchange rate constants are within the typical range accessible by FEXI (5-11,13). Standard AXR data analysis was carried out according to (6-8). To determine the ADC in the limit of low $b$ value shown in Figure $4 \mathrm{~b}$, the maximum detection $b$ value was adjusted for different $D^{\text {eq }}$ to $0.1 / D^{\text {eq }}$. Just as in other simulations, eight logarithmically spaced $b$ values were also used in this case. To detect the slice thickness threshold (Fig. 5b), the slice thickness was stepped by $0.1 \mathrm{~mm}$ in the range of 0.6 to $10 \mathrm{~mm}$ for 30 linearly spaced $D^{\text {eq }}$ values in the range of 0.207 to $2.41 \mu \mathrm{m}^{2} / \mathrm{ms}$ and 30 linearly spaced $b_{\mathrm{f}}$ values in the range of 500 to $3000 \mathrm{~s} / \mathrm{mm}^{2}$. The threshold value was set to the slice thickness yielding $\mathrm{AXR} / \mathrm{k}$ closest to 0.75 .

\section{RESULTS}

The effect of butterfly gradients in FEXI is illustrated in Figure 1c using theoretical calculations. At long mixing times, the non-filtered and filtered $\operatorname{ADC}\left(t_{\mathrm{m}}\right)$ converge to the common value of $\mathrm{ADC}_{\infty}$, which is reduced compared to the equilibrium $\mathrm{ADC}^{\mathrm{eq}}$ as a result of the butterfly gradients. This reduction is proportional to the ratio $q_{\mathrm{b}}^{2} / k$ according to Eq. [5].

The effect of increasing the crusher gradient in experiments on yeast suspension is shown in Figure 2. It can be observed that at a given $t_{\mathrm{m}}$, both non-filtered (circles, dashed lines) and filtered (asterisks, solid lines) ADC decrease with increasing crusher gradient strength as expected from the simulations in Figure 1c. The filtered ADC, respectively, increases or decreases with $t_{\mathrm{m}}$ for low or high crusher gradient strengths.

The predicted AXR bias caused by the butterfly gradients can be inspected in Figure 3, where the ratio AXR/k is shown as a function of slice thickness. The minimum required dephasing magnitude $q_{\mathrm{b}}$ was used for each slice thickness, given by Eq. [6]. The horizontal dashed lines indicate the bias for infinite slices, corresponding to $q_{\mathrm{b}}=0$. The underestimation of AXR is more pronounced at lower $k$ (blue lines in Fig. 3), which echoes the bias of $\mathrm{ADC}_{\infty}$ in Eq. [5] and is illustrated in Figure 1c. Figure 3 indicates that the AXR bias is expected to rapidly increase when reducing the slice thickness below $2.5 \mathrm{~mm}$.

The AXR/ $k$ bias may, in addition to the slice thickness, depend on other system parameters such as the fraction of extracellular water, $b_{\mathrm{f}}$, and the apparent diffusivities in the extracellular and intracellular compartments, the latter depending on the restriction size. In the example presented here (Fig. 3), the maximum AXR/k bias (at $k=1 \mathrm{~s}^{-1}$ ) for slices of $2.5 \mathrm{~mm}$ is approximately $-12 \%$ (i.e., AXR is underestimated by $12 \%$ ). A slightly increased bias of $-15 \%$ is obtained when the fast diffusing fraction $f_{1}$ increases from 0.5 to 0.8 . Considering spherical restrictions with diameters in the range of 5 to $10 \mu \mathrm{m}, f_{1}=0.5$, and slices of $2.5 \mathrm{~mm}$,

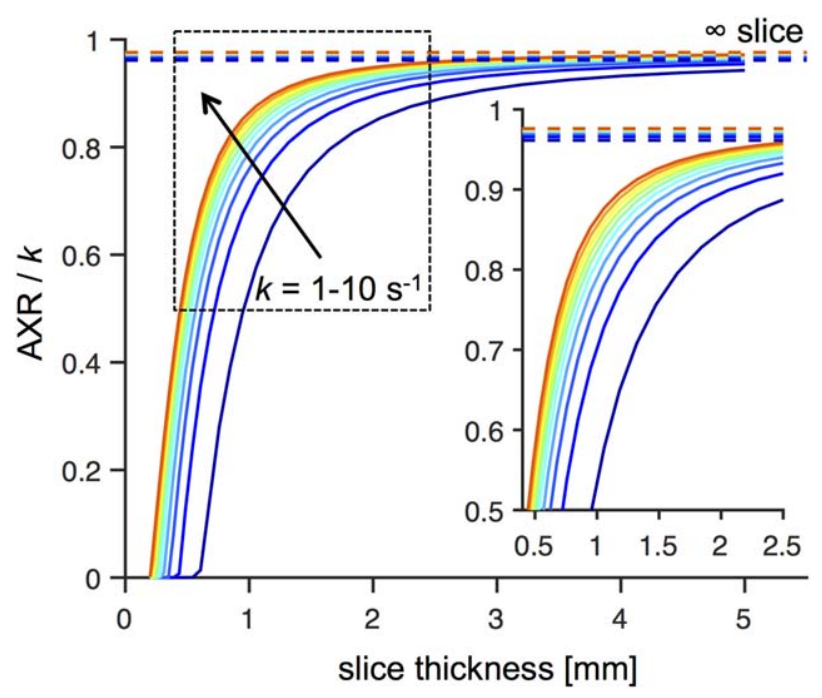

FIG. 3. Simulated bias of $A X R / k$ versus slice thickness $\Delta z$. Shown are the results of the standard AXR analysis (6) applied on noiseless synthetic data generated by Eqs. [1-4]. The simulation parameters were $b_{\mathrm{f}}=1000 \mathrm{~s} / \mathrm{mm}^{2}$ and $D^{\mathrm{eq}}=0.69 \mu \mathrm{m}^{2} / \mathrm{ms}$. Additional information is provided in the methods section. At a given slice thickness, the minimum required dephasing magnitude $q_{\mathrm{b}}$ was used according to Eq. [6]. Dashed lines show the asymptotic value for infinitely thick slices corresponding to $q_{b}=0$. The negative bias of $A X R / k$ increases for lower exchange rate constants. The inset shows the zoomed region outlined by the dashed rectangle. 

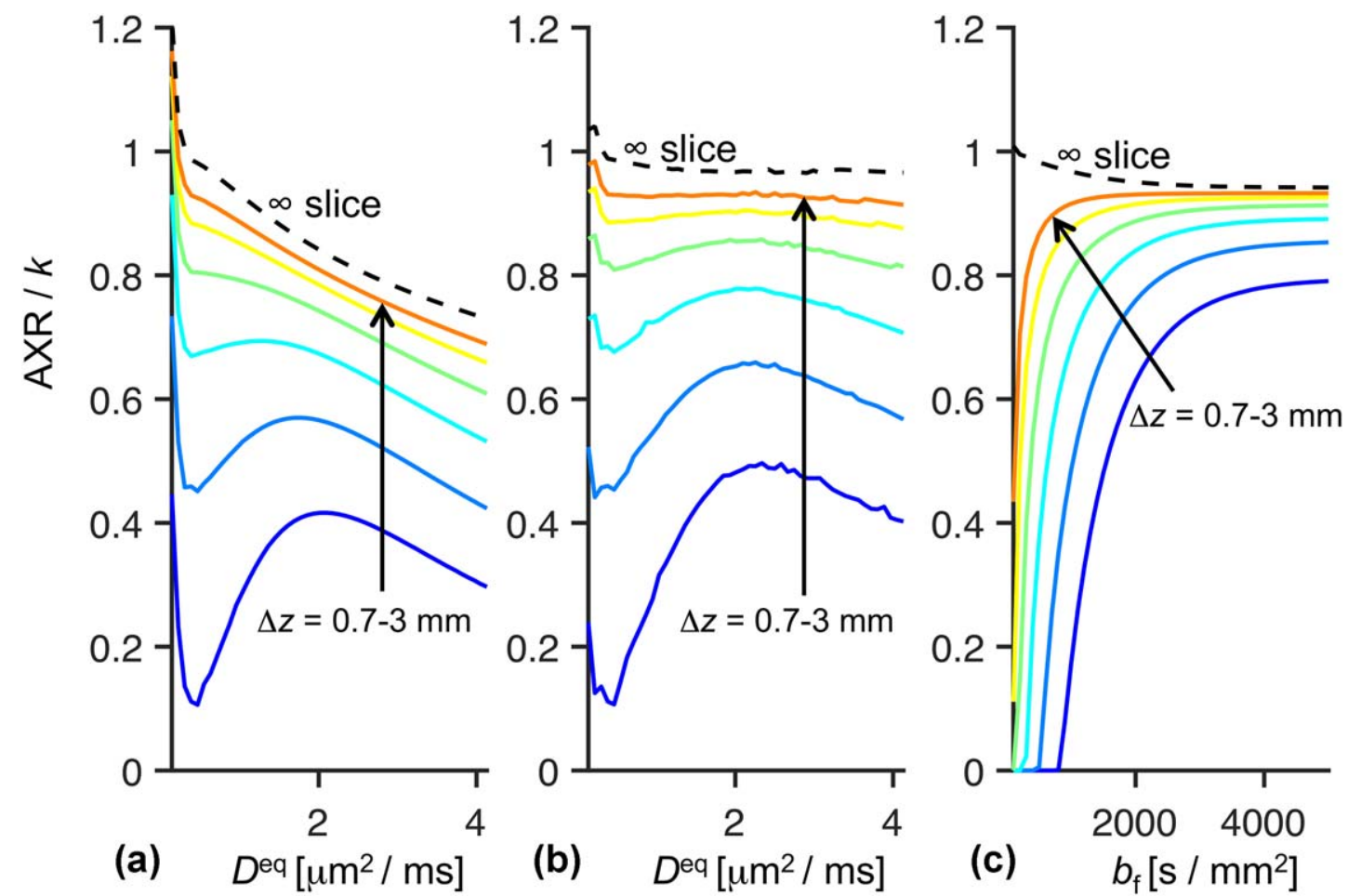

FIG. 4. Simulated bias of $A X R / k$ vs. $D^{e q}, b_{f}$, and $\Delta z$. Shown are results of the $A X R$ analysis similar to Figure 3 for $k=1 \mathrm{~s}^{-1}$ and six slices with thickness logarithmically spaced in the range $\Delta z=0.7-3 \mathrm{~mm}$. The same ratio of compartment diffusivities and signal fraction as in Figure 3 were used. Dashed lines show the asymptotic value for infinitely thick slices corresponding to $q_{\mathrm{b}}=0$. In (a) and (b), $b_{\mathrm{f}}=1000 \mathrm{~s} / \mathrm{mm}^{2}$ and in (c), $D^{\text {eq }}=0.69 \mu \mathrm{m}^{2} / \mathrm{ms}$. In (a) and (c), the same detection $b$ values as in Figure 3 were used. In (b), the maximum detection $b$ value was adjusted for different $D^{\text {eq }}$ to $0.1 / D^{\text {eq }}$.

calculations yield a similar AXR/ $k$ bias of approximately $-15 \%$.

Examining the dependence of the $\mathrm{AXR} / \mathrm{k}$ bias as a function of $D^{\mathrm{eq}}$ (at $k=1 \mathrm{~s}^{-1}$ and $b_{\mathrm{f}}=1000 \mathrm{~s} / \mathrm{mm}^{2}$ ) shows a monotonically increasing bias with increasing $D^{\text {eq }}$ for thicker slices and an alternating trend for thinner slices (Figs. 4a, b). This alternating trend can be explained with the results in Figure 4c, which shows a decreasing bias with increasing $b_{\mathrm{f}}$ (at $k=1 \mathrm{~s}^{-1}$ and $D^{\mathrm{eq}}=0.69 \mu \mathrm{m}^{2}$ / $\mathrm{ms})$. The effect of using larger or smaller detection $b$ values is visible by comparing Figures $4 \mathrm{a}$ and $4 \mathrm{~b}$, respectively. The AXR/ $k$ bias is strongly dependent on slice thickness but is also modulated by $D^{\mathrm{eq}}$ and $b_{\mathrm{f}}$. This modulation can be further inspected in Figure 5a for slices of $2.5 \mathrm{~mm}$ and in Figure $5 \mathrm{~b}$ showing the slice thickness threshold above which the bias is $<25 \%$.

\section{DISCUSSION}

Our results indicate that in FEXI (Fig. 1) imaging gradients are expected to cause biased AXR results when thin imaging slices are used. In FEXI, the time course of the filtered $\operatorname{ADC}\left(t_{\mathrm{m}}\right)$ is used to quantify AXR (6) (Fig. 1c). In the AXR model, the filtered $\operatorname{ADC}\left(t_{m}\right)$ is expected to exponentially approach the equilibrium $\mathrm{ADC}^{\mathrm{eq}}$ at long mixing times $t_{\mathrm{m}}(6)$. The $\mathrm{ADC}^{\mathrm{eq}}$ is estimated from the signal attenuation at short $t_{\mathrm{m}}$ and without diffusion filter. The sensitivity of $\mathrm{ADC}^{\mathrm{eq}}$ to the butterfly gradients is therefore negligibly small. However, this is not the case for $\mathrm{ADC}_{\infty}$, which is the non-filtered ADC measured at long mixing times. The diffusion weighting by the butterfly gradients results in additional modulation of the $\operatorname{ADC}\left(t_{\mathrm{m}}\right)$, which can be understood as an extended lowpass diffusion filtering effect during the mixing interval $t_{\mathrm{m}}$. According to Eq. [5], the extended filtering of the fast diffusion component reduces the $\mathrm{ADC}_{\infty}$. This effect increases at low exchange rates.

As discussed in $(7,8)$, the reduced asymptotic $\mathrm{ADC}_{\infty}$ in FEXI could be a consequence of multiple compartments with distinctly different exchange rates, where a slow exchange component could not be detected despite having long $t_{\mathrm{m}}$. However, our calculations (Fig. 1c) and experiments (Fig. 2) indicate that a reduced $\mathrm{ADC}_{\infty}$ could also be caused by the butterfly gradients. The reduced $\mathrm{ADC}_{\infty}$ may therefore indicate that too large butterfly gradients were applied. Note that $\mathrm{ADC}_{\infty}$ is not measured in a typical FEXI protocol. To ensure that reliable FEXI data is acquired, which is not significantly affected by butterfly gradients, it is therefore recommended to measure the non-filtered ADC at short as well as at long $t_{\mathrm{m}}$. The experimental results in Figure 2 illustrate the effect of increasing $q_{\mathrm{b}}$ exceeding the minimum requirement for 3 -mm slices, which is $4.2 \mathrm{~mm}^{-1}$. The bias at $q_{\mathrm{b} \text {,min }}$ for various slice thicknesses can be inferred from Figure 2 using Eq. [6]. The slight increase of the non-filtered ADC observed in Figure 2 at low crusher gradients (light brown dashed line) could be because of longitudinal relaxation differences between the intra- and extracellular compartments, similar to the effect observed in spinecho sequences in the presence of transverse relaxation 

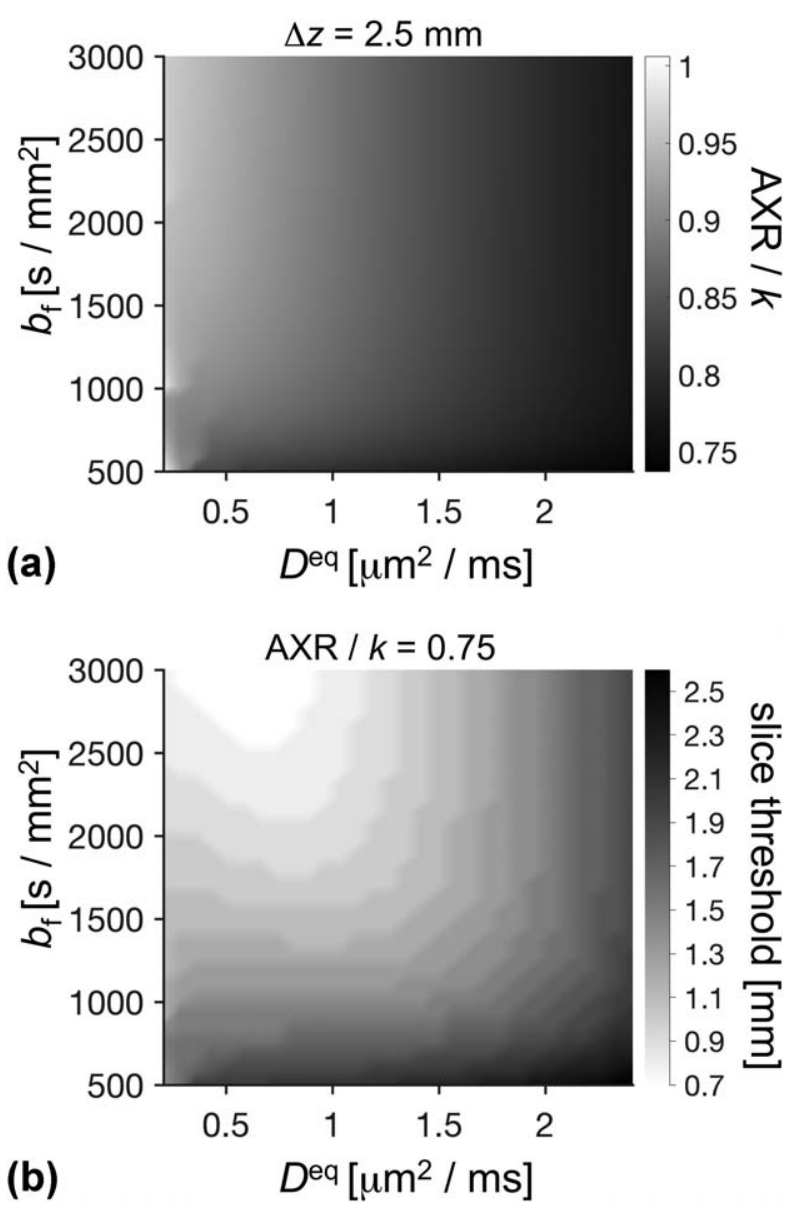

FIG. 5. Bias of $A X R / k$ and the slice thickness threshold as a function of $D^{\text {eq }}$ and $b_{\mathrm{f}}$. (a) Contour plots of $\mathrm{AXR} / \mathrm{k}$ for a $2.5 \mathrm{~mm}$ slice. (b) Slice thickness threshold, above which the AXR/k bias is less than $25 \%$. The simulation is carried out similarly as in Figures 3 and 4 for $k=1 \mathrm{~s}^{-1}$. The increasingly darker shade indicates an increasing $A X R / k$ bias (a) or an increasing slice thickness threshold (b). Thinner slices "are allowed" in brighter regions.

differences (33-36). Quantitative assessment of relaxation was beyond the scope of this work, but it could be estimated by relaxation-diffusion correlation experiments $(1,2)$ or by an extended FEXI protocol using complementary relaxation-diffusion correlation experiments (37). A monotonic decrease of the filtered ADC observed at high $q_{\mathrm{b}}$ in Figure 2 (black solid line) is caused by the exceedingly strong $q_{\mathrm{b}}$, providing diffusion filtering during $t_{\mathrm{m}}$ that overshadows the effect of the filter block, $q_{\mathrm{f}}$. This effect is predicted also by calculations shown in Figure 1c.

As shown in Figure 3, the butterfly gradients lead to underestimation of AXR. The AXR bias increases for thinner slices associated with stronger butterfly gradients. The small negative bias observed for large slices is consistent with previously reported simulations inspecting the AXR bias as a function of filter strength, $b_{\mathrm{f}}$, and SNR (6). This bias originates from fitting the AXR model (6) to the data from a limited range of $t_{\mathrm{m}}$ values. In Figure 3, the AXR bias is more pronounced at lower $k$. Again, this observation can be explained in terms of an increased low-pass diffusion filtering effect at low $k$. Most importantly, our results suggest that the AXR bias depends on slice thickness in a strongly non-linear fashion. This suggests that for $b_{\mathrm{f}}=$ $1000 \mathrm{~s} / \mathrm{mm}^{2}$ and $D^{\mathrm{eq}}=0.69 \mu \mathrm{m}^{2} / \mathrm{ms}$, the AXR bias can be considered negligible $(<15 \%)$ above a slice thickness threshold of approximately $2.5 \mathrm{~mm}$.

Although the abrupt increase in the AXR bias is characteristic for any $b_{\mathrm{f}}$ and $D^{\mathrm{eq}}$ value, the slice thickness threshold varies. The AXR bias as a function of $D^{\text {eq }}$ (proportional increase of $D_{1}$ and $D_{2}$ ) is shown in Figure 4a. The initial increase of the AXR bias (reduced AXR/k), which is more pronounced for thinner slices, is caused by the increasing effect of the imaging gradients when the efficiency of the FEXI diffusion filter is very low. With increasing $D^{\mathrm{eq}}$, the filter efficiency increases up to a point of saturation, resulting in a decrease of AXR bias (increase of AXR/k). For larger $D^{\mathrm{eq}}$, the AXR bias increases again (decrease of AXR/k). The increase of the AXR bias observed for infinite slices (dashed line) (i.e., at $q_{\mathrm{b}}=0$ ) is related to the biased estimation of ADC using a finite range of detection $b$ values. This effect can be eliminated if the range of $b$ values is adjusted to yield maximum attenuation of 0.1 at every $D^{\text {eq }}$ (see Fig. $4 \mathrm{~b}$ ). The reduction of the AXR bias as a function of $b_{\mathrm{f}}$ is illustrated in Figure $4 \mathrm{c}$ for $D^{\mathrm{eq}}=0.69 \mu \mathrm{m}^{2} / \mathrm{ms}$.

Which slice thicknesses can be safely applied to avoid the AXR bias? The answer depends on tissue properties and experimental parameters. The key parameters for predicting the AXR bias are $D^{\mathrm{eq}}$ and $b_{\mathrm{f}}$. The contour plot of $\mathrm{AXR} / \mathrm{k}$ for a slice of $2.5 \mathrm{~mm}$ is shown in Figure 5a. In Figure 5b, we examined the slice thickness threshold, above which the bias is $<25 \%$. Figure 5 can be used to roughly estimate the optimal $b_{\mathrm{f}}$ for a known $D^{\mathrm{eq}}$ or infer the expected bias when $k=1 \mathrm{~s}^{-1}$. The maps are qualitatively similar for different values of $k$ as can be inferred from Figure 3. The brighter areas in Figure 5 indicate regions with lower bias or regions where thinner slices can be used.

The presented analysis assumes Gaussian or timeindependent diffusion in both extracellular and intracellular compartments. Therefore, the question arises whether restricted diffusion would affect conclusions drawn from such a simplified analysis. In case of slow exchange, effects of restricted diffusion can be included in the analysis by considering time-dependent diffusion as in (23). For time-dependent diffusion, the signal evolution can also be calculated numerically using Eq. [1], which needs to be segmented into shorter time intervals, during which the diffusion matrix in Eq. [4] can be considered constant. In each time interval, the time-dependent diffusion $D(t)$ should then be used. The Gaussian phase distribution approximation for the time-dependent apparent diffusivity in various restriction geometries, $D(\delta, t)$, which account also for a finite diffusion encoding pulse length, are summarized in (31). Based on our calculations, we conclude that accounting for restricted diffusion yields qualitatively similar results (not shown) as presented in Figure 3. Considering the effects of restricted diffusion as well as the effects of varying the fraction of extracellular water $f_{1}$, we conclude that neither of these conditions significantly affects the AXR bias for the range of parameters used in our analysis. 
Our results suggest that for total exchange rates $k \geq 1$ $\mathrm{s}^{-1}$ the butterfly gradients are not expected to cause significant AXR bias in typical clinical settings $(7,9,10)$, demanding thicker slices (of $\sim 5 \mathrm{~mm}$ ) to achieve adequate SNR levels. However, for thinner slices (of $\sim 1.5-3 \mathrm{~mm}$ ) used in preclinical FEXI experiments $(8,11)$, the AXR bias may be significant. Our analysis therefore calls for caution and illustrates the need to keep the butterfly gradients in check. The undesired effects of butterfly gradients could be identified in pilot experiments by comparing the non-filtered ADC at short and long $t_{\mathrm{m}}$.

The imaging gradients might also inadvertently affect outcomes of other DDE experiments, particularly those using longitudinal magnetization storage. In some DDE sequences (12), short or long mixing times between two consecutive diffusion encoding blocks can be used to probe size (38) or anisotropy (39) of restrictions. Note that the term "mixing time" in DDE does not necessarily involve the longitudinal storage. In DDE, the longitudinal storage is usually implemented only when diffusionweighted STE is used (40). When diffusion weighting is desired during the longitudinal storage, as in the case of an STE sequence used for DTI, the effects of the imaging gradients can easily be compensated (17). However, in situations where diffusion weighting is not desired during the longitudinal storage, the undesired effects of imaging gradients cannot be easily mitigated. Possible sequence modifications might involve using RF phase-cycling $(4,5,15)$. Alternatively, an increased resolution might be achieved by combining acquisitions with overlapping slices and super-resolution reconstruction techniques (41). To minimize the undesired diffusion weighting during the mixing interval in FEXI, slice selection could be omitted from the filter block and implemented only in the detection block (13). However, such implementation still requires to properly filter magnetization coherences either by phasecycling of the RF pulses or by using crusher gradients as shown in Figure 1. Because it has not been reported, we presume that in (13), coherences were filtered by phasecycling. The bias caused by the crusher gradients alone would be reduced because of the lower required dephasing magnitude according to Eq. [6], yielding $q_{\mathrm{b}, \min }=4 \pi / \Delta z$. In all our simulation results, the slice thickness would in this case need to be rescaled by a factor of approximately $4 / 7$.

\section{CONCLUSIONS}

In FEXI experiments on a yeast cell suspension, we observed the undesired effects of additional diffusion weighting caused by the butterfly gradients during the longitudinal storage interval. To examine the AXR bias caused by the butterfly gradients as a function of slice thickness, a two-compartment system signal evolution was calculated using matrix exponential operators for each segment (block) of the FEXI pulse sequence.

Because of the butterfly gradients, reducing the slice thickness inevitably leads to increased diffusion weighting during $t_{\mathrm{m}}$, hence resulting in an increased underestimation of the AXR. The AXR bias is more pronounced at lower exchange rates, which could be understood in terms of the efficiency of the low-pass diffusion filtering during $t_{\mathrm{m}}$. Analysis of the AXR bias as a function of slice thickness reveals a threshold under which the AXR underestimation abruptly increases. The bias can therefore be avoided by using sufficiently large slices. In typical clinical FEXI requiring slices of approximately $5 \mathrm{~mm}$ to achieve adequate SNR, the AXR bias is expected to be negligible for $k \geq 1 \mathrm{~s}^{-1}$. However, the bias may become significant in preclinical experiments where thinner slices are used. The AXR bias is also sensitive to the choice of the $b_{\mathrm{f}}$ value and affected by the mean diffusivity $D^{\mathrm{eq}}$. The bias may also become significant in samples or tissue with larger diffusivities.

The confounding effect of butterfly gradients, demonstrated here for the case of FEXI, may be critical in any sequence with multiple encodings and variable duration of the longitudinal storage. Alternative sequence designs allowing to circumvent or account for the confounding effects of butterfly gradients would be of great importance in correlation experiments such as FEXI. Using slice-selective pulses only in the detection block might be a viable alternative to reduce the AXR bias.

\section{ACKNOWLEDGMENTS}

This project was supported by the Danish Multiple Sclerosis Society and the Capital Region Research foundation for Healthcare, both with T.B.D. as principal investigator (PI). H.L. is supported by a Sapere Aude award from the Danish Research Council for Independent Research, Production and Technology.

\section{REFERENCES}

1. Bernin D, Topgaard D. NMR diffusion and relaxation correlation methods: new insights in heterogeneous materials. Curr Opin Colloid Interface Sci 2013;18:166-172.

2. Galvosas P, Callaghan PT. Multi-dimensional inverse Laplace spectroscopy in the NMR of porous media. C R Phys 2010;11:172-180.

3. Lee J, Labadie C, Springer CS, Harbison GS. Two-dimensional inverse Laplace transform NMR: altered relaxation times allow detection of exchange correlation. J Am Chem Soc 1993;115:7761-7764.

4. Callaghan PT, Furó I. Diffusion-diffusion correlation and exchange as a signature for local order and dynamics. J Chem Phys 2004;120:40324038.

5. Åslund I, Nowacka A, Nilsson M, Topgaard D. Filter-exchange PGSE NMR determination of cell membrane permeability. J Magn Reson 2009;200:291-295.

6. Lasič S, Nilsson M, Lätt J, Ståhlberg F, Topgaard D. Apparent exchange rate mapping with diffusion MRI. Magn Reson Med 2011;66:356-365.

7. Nilsson M, Lätt J, van Westen D, Brockstedt S, Lasič S, Ståhlberg F, Topgaard D. Noninvasive mapping of water diffusional exchange in the human brain using filter-exchange imaging. Magn Reson Med 2013;69:1572-1580.

8. Sønderby CK, Lundell HM, Søgaard LV, Dyrby TB. Apparent exchange rate imaging in anisotropic systems. Magn Reson Med 2014;72:756762.

9. Lampinen B, Szczepankiewicz F, van Westen D, Englund E, C Sundgren P, Lätt J, Ståhlberg F, Nilsson M. Optimal experimental design for filter exchange imaging: apparent exchange rate measurements in the healthy brain and in intracranial tumors. Magn Reson Med 2017;77:1104-1114.

10. Lasič S, Oredsson S, Partridge SC, Saal LH, Topgaard D, Nilsson M, Bryshke K. Apparent exchange rate for breast cancer characterization. NMR Biomed 2016;29:631-639.

11. Schilling F, Ros S, Hu DE, et al. MRI measurements of reportermediated increases in transmembrane water exchange enable detection of a gene reporter. Nat Biotechnol 2016;35:75-80.

12. Shemesh N, Jespersen SN, Alexander DC, et al. Conventions and nomenclature for double diffusion encoding NMR and MRI. Magn Reson Med 2016;75:82-87. 
13. Tian X, Li H, Jiang X, Xie J, Gore JC, Xu J. Evaluation and comparison of diffusion MR methods for measuring apparent transcytolemmal water exchange rate constant. J Magn Reson 2017;275:29-37.

14. Price W. NMR Studies of translational motion: principles and applications. Cambridge: Cambridge University Press; 2009. 416 p.

15. Khrapitchev AA, Callaghan PT. Double PGSE NMR with stimulated echoes: phase cycles for the selection of desired encoding. J Magn Reson 2001;152:259-268.

16. Bernstein M, King K, Zhou X. Handbook of MRI pulse sequences. Cambridge: Elsevier; 2004. 1040 p.

17. Lundell H, Alexander DC, Dyrby TB. High angular resolution diffusion imaging with stimulated echoes: compensation and correction in experiment design and analysis. NMR Biomed 2014;27:918-925.

18. Sigmund EE, Novikov DS, Sui D, et al. Time-dependent diffusion in skeletal muscle with the random permeable barrier model (RPBM): application to normal controls and chronic exertional compartment syndrome patients. NMR Biomed 2014;27:519-528.

19. Zimmerman JR, Brittin WE. Nuclear magnetic resonance studies in multiple phase systems: lifetime of a water molecule in an adsorbing phase on silica gel. J Phys Chem 1957;61:1328-1333.

20. Allan EA, Hogben MG, Shaw KN. Multi-site chemical exchange by NMR. Pure Appl Chem 1972;32:9-26.

21. Kärger J. NMR self-diffusion studies in heterogeneous systems. Adv Colloid Interface Sci 1985;23:129-148.

22. Andrasko J. Water diffusion permeability of human erythrocytes studied by a pulsed gradient NMR technique. Biochim Biophys Acta 1976;428:304-311.

23. Stanisz GJ, Szafer A, Wright GA, Henkelman RM. An analytical model of restricted diffusion in bovine optic nerve. Magn Reson Med 1997;37:103-111.

24. Pfeuffer J, Flögel U, Dreher W, Leibfritz D. Restricted diffusion and exchange of intracellular water: theoretical modelling and diffusion time dependence of $1 \mathrm{H}$ NMR measurements on perfused glial cells. NMR Biomed 1998;11:19-31.

25. Price WS, Barzykin A V, Hayamizu K, Tachiya M. A model for diffusive transport through a spherical interface probed by pulsed-field gradient NMR. Biophys J 1998;74:2259-2271.

26. Davoodi-Bojd E, Chopp M, Soltanian-Zadeh H, Wang S, Ding G, Jiang Q. An analytical model for estimating water exchange rate in white matter using diffusion MRI. PLoS One 2014;9:e95921.

27. Schmidt-Rohr K, Spiess HW. Multidimensional solid-state NMR and polymers. Cambridge: Elsevier; 2012. 496 p.
28. Van Landeghem M, Haber A, D’espinose De Lacaillerie JB, Blümich B. Analysis of multisite 2D relaxation exchange NMR. Concepts Magn Reson Part A 2010;36:153-169.

29. Valette J, Lethimonnier F, Lebon V. About the origins of NMR diffusionweighting induced by frequency-swept pulses. J Magn Reson 2010;205: 255-259.

30. Topgaard D, Sakellariou D. Diffusion damping during adiabatic zrotation pulses for NMR spectroscopy in inhomogeneous magnetic fields. J Chem Phys 2006;125:44503.

31. Åslund I, Topgaard D. Determination of the self-diffusion coefficient of intracellular water using PGSE NMR with variable gradient pulse length. J Magn Reson 2009;201:250-254.

32. Murday JS, Cotts RM. Self-diffusion coefficient of liquid lithium. J Chem Phys 1968;48:4938.

33. Schönhoff M, Söderman O. PFG-NMR diffusion as a method to investigate the equilibrium adsorption dynamics of surfactants at the solid/liquid interface. J Phys Chem B 1997;101:8237-8242.

34. Li H, Jiang X, Xie J, Gore JC, Xu J. Impact of transcytolemmal water exchange on estimates of tissue microstructural properties derived from diffusion MRI. Magn Reson Med 2017;77:2239-2249.

35. Peled S, Cory DG, Raymond SA, Kirschner DA, Jolesz FA. Water diffusion, T2, and compartmentation in frog sciatic nerve. Magn Reson Med 1999;42:911-918.

36. Branzoli F, Ercan E, Webb A, Ronen I. The interaction between apparent diffusion coefficients and transverse relaxation rates of human brain metabolites and water studied by diffusion-weighted spectroscopy at $7 \mathrm{~T}$. NMR Biomed 2014;27:495-506.

37. Eriksson S, Elbing K, Söderman O, Lindkvist-Petersson K, Topgaard D, Lasič S. NMR quantification of diffusional exchange in cell suspensions with relaxation rate differences between intra and extracellular compartments. PLoS One 2017;12:1-18.

38. Mitra P. Multiple wave-vector extensions of the NMR pulsed-fieldgradient spin-echo diffusion measurement. Phys Rev B Condens Matter 1995;51:15074-15078.

39. Jespersen SN, Lundell H, Sønderby CK, Dyrby TB. Orientationally invariant metrics of apparent compartment eccentricity from double pulsed field gradient diffusion experiments. NMR Biomed 2013;26:1647-1662.

40. Cheng Y, Cory DG. Multiple scattering by NMR. J Am Chem Soc 1999;121:7935-7936.

41. Ning L, Setsompop K, Michailovich O, Makris N, Shenton ME, Westin CF, Rathi Y. A joint compressed-sensing and super-resolution approach for very high-resolution diffusion imaging. Neuroimage 2016;125:386-400. 